\title{
An MILP Approach to Cancer Chemotherapy Dose Regime Design
}

\author{
John M. Harrold and Robert S. Parker \\ Department of Chemical and Petroleum Engineering \\ 1249 Benedum Hall, Pittsburgh PA, 15261 \\ rparker@pitt.edu
}

\begin{abstract}
Cancer chemotherapy is a form of treatment in which both healthy and diseased tissues are adversely affected by dosing; this requires the clinician make decisions which balance the effectiveness of treatment with toxicity effects, financial, and logistical constraints. This paper presents a model-based approach for chemotherapy treatment scheduling that accounts for both drug toxicity constraints and the dosing constraints associated with clinical practice. A mixed-integer linear programming (MILP) approach is applied to a nonlinear system considered previously in an optimal control framework by Martin and Teo [1]. The nonlinearities in the pharmacodynamic PD model were eliminated by logarithmic transform and both the pharmacokinetic (PK) and PD models were converted into algebraic constraints by discretizing the system over the treatment window. This formulation also accounts for the toxicity constraints and allows for the easy incorporation of dosing constraints which would be encountered during the course of treatment. MILP results are shown for three cases considered in [1]: highly effective drug, moderately effective drug, and a drug with very little effect on the tumor. In the limit of the discretization timestep and delivery interval approaches zero, the MILP algorithm returns the optimal control solution. Like the optimal control solution, most of the drug is delivered at the end of the treatment cycle, which results in the minimal final cancer cell population. The MILP approach results in a problem which can be solved to optimality and can easily be augmented with constraints which give the problem more clinical relevance.
\end{abstract}

\section{Introduction}

Collectively cancer represents a set of diseases in which a disturbance has occurred in the homeostatic balance between apoptosis, or programmed cell death, and normal cellular proliferation [2]. Several techniques are employed clinically to treat cancer: surgical extraction of tumor mass, immunotherapy, chemotherapy, and radiation, among others. Cancerous cells prolifer- ate more rapidly than normal cells, and consequently they traverse through the cell-cycle more frequently [3]. Chemotherapy is a popular method of treatment which attempts to selectively target cancerous cells by taking advantage of the frequent transitions through the cell cycle. Complete selectivity of diseased tissues over healthy tissues is rarely attained; consequently, chemotherapy normally affects both healthy and diseased tissues [4].

Chemotherapeutic treatment of cancer is a dichotomy: eliminate the disease while simultaneously minimizing the side effects to healthy tissues. Clinically, the development of drug delivery schedules is an empirical process which has been established through experience [5]. The schedules developed in he present work are capable of considering drug efficacy, issues associated with toxicity and what will be termed "clinical constraints" in a single formulation. Clinical constraints include both logistical and economic issues associated with patient treatment. Instructing patients to take drugs too frequently or at odd times can result in patient adherence problems [6]. Schedules requiring extended hospital stays can also become prohibitively expensive. Consequently a drug delivery schedule which can balance the need for efficacy with side effects, while simultaneously accounting for clinically-relevant constraints, would be a valuable tool for clinicians.

In this work we establish a mixed-integer linear programming (MILP) approach to the development of optimal drug delivery profiles. This algorithm accounts for the opposing objectives of disease treatment and toxicity minimization, where the latter is characterized by constraints associated with treatment dose and exposure. Previous approaches to this class of problems typically employ optimal control techniques [7-11]. While this methodology provides mathematically optimal solutions, they are most likely not optimal from a clinical perspective for the financial and logistical reasons discussed above. As a case study, the present work revisits the cancer treatment problem initially posed in [1]; a reformulation of the control problem within an MILP framework is presented here. This allows for the 
inclusion of binary decision variables to account for discontinuities associated with drug effects, which can be extended to provide a flexible way of incorporating constraints that can represent the realities of clinical treatment.

\section{Cancer Chemotherapy Modeling}

Model based control requires a mathematical description of the system capable of accurately representing the system dynamics $[12,13]$. Cancer chemotherapy modeling is typically segregated into drug pharmacokinetics $(\mathrm{PK})$, describing how the drug distributes throughout an organism, and pharmacodynamics (PD), which delineates cellular proliferation dynamics, and the way the drug effects the organism. PK models are normally used to predict the drug plasma concentration in response to drug administration. Predictions of plasma concentrations are important for two reasons. The first is that many toxicity metrics are derived from plasma drug exposure. Secondly, it is both invasive and expensive to determine the drug concentration in tumors, therefore drug plasma concentrations are assumed to represent the concentration of the drug at the site of the cancer [1].

\subsection{Pharmacokinetics}

PK models predicting plasma concentrations tie the drug administration to the effects which are manifested in the reduction of the cancerous burden. Physiological understanding ultimately limits the complexity of a PK model. For drugs administered intravenously (iv), a mass balance over the plasma, commonly referred to as a one compartment model [14], can be used to describe the PK:

$$
\begin{aligned}
\dot{v}(t) & =u(t)-\gamma v(t) \\
v(0) & =v_{0}
\end{aligned}
$$

With an initial drug concentration of $v_{0}$, the PK model (1) quantifies the change in drug concentration with respect to the amount of drug administered, $u(t)$, and metabolic elimination at a rate $\gamma$.

\subsection{Tumor Growth Modeling}

PD modeling here considers cancer in the form of a solid tumor and is built upon models representing untreated tumor proliferation [15]. Three different models have been suggested to account for tumor proliferation in the absence of treatment [1]: exponential, logistic, and Gompertzian (given by equations (3a), (3b), and (3c), respectively).

$$
\begin{aligned}
& \dot{N}_{e}=\frac{\ln 2}{\tau} N_{e} \\
& \dot{N}_{l}=\frac{1}{\tau} \ln \left[\frac{\theta-N_{0}}{\theta / 2-N_{0}}\right] N_{l}\left[1-\frac{N_{l}}{\theta}\right] \\
& \dot{N}_{g}=\frac{1}{\tau} \ln \left[\frac{\ln \left(\theta / N_{0}\right)}{\ln \left(\theta / 2 N_{0}\right)}\right] N_{g} \ln \left[\frac{\theta}{N_{g}}\right]
\end{aligned}
$$

Figure 1 shows the models from equation (3) on a loglinear scale. Tumor masses become detectable at approximately $10^{9}$ cells [16], while multiplying at an exponential rate. This characteristic log-linear growth can be seen at the early times in Figure 1. The exponential model (3a) assumes this proliferation is constant with a doubling time of $\tau$. Many tumor masses however experience reduced rates of proliferation as they approach a plateau population $\theta$. In Figure 1, the plateau population of $10^{12}$ cells was used. This is approximately one $\mathrm{kg}$ of cells, enough to kill most patients [16]. It is common for the plateau population to be significantly larger than $10^{12}$ cells [15], meaning the exponential model is sufficient to describe cellular proliferation. When the plateau population is at or around $10^{12}$ the logistic (3b) and Gompertzian (3c) models account for the observed decreases in proliferation rates [17]. Both models (3b) and $(3 \mathrm{c})$ asymptotically approach $\theta$.

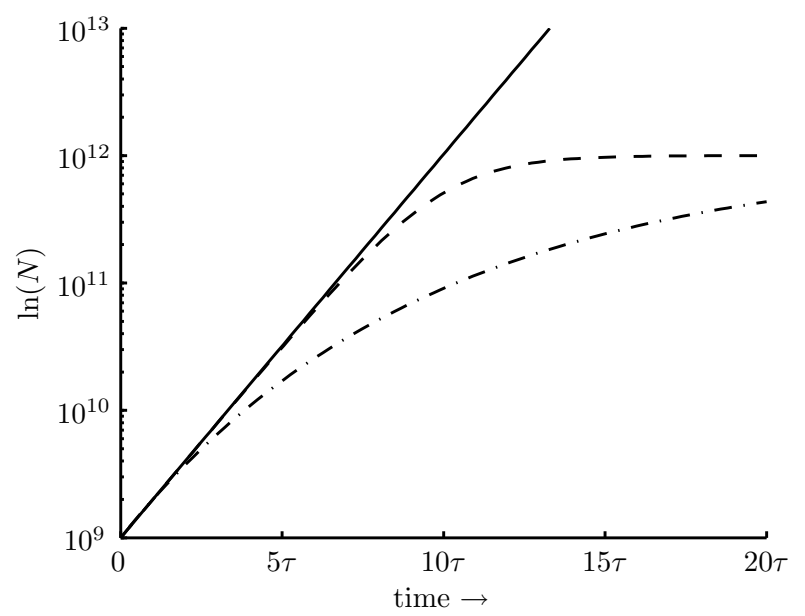

Figure 1: Uncontrolled tumor growth models $[1,15]$ : exponential (solid), logistic (dashed), Gompertz (dash-dot). $\left(\tau=2\right.$ time units, $N_{0}=10^{9}$ cells, and $\theta=10^{12}$ cells)

\subsection{Pharmacodynamics}

The PD effect model for cancer cells proliferating in a Gompertzian fashion (3c) with an $i v$ administered drug can be modeled using equations (1) and (4) [1].

$$
\begin{aligned}
\dot{N(t)=} & \frac{1}{\tau} \ln \left[\frac{\ln \left(\theta / N_{0}\right)}{\ln \left(\theta / 2 N_{0}\right)}\right] N(t) \ln \left[\frac{\theta}{N(t)}\right] \\
& -k_{d}\left(v(t)-v_{t h}\right) \mathcal{H}\left(v(t)-v_{t h}\right) N(t) \\
N(0)= & N_{0}
\end{aligned}
$$


By considering the tumor to grow in a homogeneous lumped fashion, this model ignores any cycle-specific effects of the drug [18]. Cancer cells (initially at $N_{0}$ ) proliferate according to (4). Gompertzian growth, the first term on the right hand side of (4), is inhibited by the presence of the drug in the kill term, the final term in (4). The rate of cell kill is proportional with constant, $k_{d}$, to the size of the tumor, $N$, and the plasma concentration of the drug, $v$, above a therapeutic concentration, $v_{t h}$. Plasma concentrations below $v_{t h}$ have no clinical benefit.

\subsection{Constraints}

2.4.1 Toxicity: To maintain efficacy while preventing unnecessary side effects, the following constraints must be satisfied during treatment:

$$
\begin{aligned}
v(t) & \leq v_{\max } ; \quad \forall t \\
\int_{0}^{t_{f}} v(t) d t & \leq v_{\text {cum }}
\end{aligned}
$$

The acute toxicity limit, (5), restricts the maximum amount of the drug which can be found in the plasma to $v_{\max }$. Integrated exposure over the duration of treatment must be less than the cumulative toxicity limits, $v_{\text {cum }}$, the maximum total amount of drug the patient can be exposed to over the treatment cycle $\left[0, t_{f}\right]$.

2.4.2 Dosing: This case study considers a drug which is administered by $i v$ infusion in the form of a bolus. Cancer chemotherapeutics administered in this fashion are often delivered over a range $\left[u_{\min }, u_{\max }\right]$. The lower bound is usually determined by the rate of drug elimination, $\gamma$, and the upper bound is set by the acute toxicity constraints of the drug.

\section{Control Problem Formulation}

\subsection{Optimal Control Problem}

Considering the system governed by (1) and (4), $u_{i}$ represents the dose of drug given at the beginning of week $i$ over the treatment window of $d$ weeks. Accordingly the optimal control objective can be written as:

$$
\min J(u)=N\left(t_{f}\right)+\Gamma\|u\|_{2}^{2}
$$

This penalizes the tumor size at the end of the treatment cycle, $t_{f}$, and the total amount of drug administered. This optimization is constrained by the PK (1), the PD (4) the constraints (5)-(6) and the initial number of cancer cells present and the initial concentration of drug present were given by $N_{0}$ and $v_{0}$, respectively.

Martin and Teo addresses the observation that increases in drug resistance occur as the cancerous populations grow by placing an upper bound on the tumor size during treatment. The initial tumor mass is set as the maximum [1]:

$$
N(t) \leq N_{\max }=N_{0} ; \quad \forall t
$$

Considering the case where $\Gamma=0$, the optimization in (7) results in a trajectory tracking problem. Details of the solution can be found in [1].

\subsection{MILP Reformulation}

3.2.1 PD Scaling: The PD equation (4) is transformed using the following relationships:

$$
P=\ln (N) \quad \rightarrow \quad e^{P}=N \quad \rightarrow \quad \dot{N}=\dot{P} e^{P}
$$

Substituting the relationships (9) into the PD equation (4) results, after some manipulation, in the following linear ODE:

$$
\begin{aligned}
\dot{P}= & \frac{1}{\tau} \ln \left[\frac{\ln \left(\theta / N_{0}\right)}{\ln \left(\theta / 2 N_{0}\right)}\right](\ln (\theta)-P) \\
& -k_{d}\left(v(t)-v_{t h}\right) \mathcal{H}\left(v(t)-v_{t h}\right)
\end{aligned}
$$

This transform is convenient because it both scales the tumor volume (important for numerical solution) and eliminates the nonlinearities associated with proliferation and drug efficacy.

3.2.2 Objective Function: The objective function for the MILP is given as:

$$
\min J(u)=P\left(t_{f}\right)+\Gamma\|u\|_{2}^{2}
$$

Minimizing the natural log of a variable will give the same result as minimizing that variable. As in the continuous problem $\Gamma=0$ was used.

3.2.3 Dynamic Constraints: MILP reformulation requires that the dynamic constraints be represented as algebraic constraints. The variables $v_{d}(j)$ and $P_{d}(j)$ are now introduced to represent the discretized counterparts of the drug concentration $(v)$ and the log of the number of tumor cells $(P)$, respectively, at any point $j$ over the range $[1, m]$. Here $m$ is the total number of timesteps in the treatment window $\left(m=\frac{t_{f}}{h}\right)$. For the drug dynamics, represented by a linear ODE (1), discretization at a timestep $h$ is straightforward [19] and results in:

$v_{d}(j+1)=\left\{\begin{array}{cc}-\frac{1}{\gamma}\left(e^{-\gamma h}-1\right) \frac{u(i)}{h}+e^{-\gamma h} v_{d}(j) & i=h j \\ e^{-\gamma h} v_{d}(j) & i \neq h j\end{array}\right.$

Timesteps which occur when a dose can be administered $(i=h j)$ have two terms. The first term represents the mass of drug $(u(i))$ administered over the duration of the timestep $(h)$, and the second term accounts for the elimination of the drug. All other timesteps $(i \neq h j)$ have only the elimination term.

Equation (10) can be discretized using Euler's method $[20]$.

$$
\begin{aligned}
& F\left(P_{d}(j), \phi(j)\right)= \\
& \quad \frac{1}{\tau} \ln \left[\frac{\ln \left(\theta / N_{0}\right)}{\ln \left(\theta / 2 N_{0}\right)}\right]\left(\ln (\theta)-P_{d}(j)\right)-k_{d} \phi(j)
\end{aligned}
$$


The function, $F$, represents the $\mathrm{PD}$ equation and introduces the variable $\phi(j)$, which can be thought of as the effective drug concentration at the site of the cancer. The introduction of $\phi(j)$ is necessary to address the discontinuity associated with drug effect. The binary variable $b(j)$ allows $\phi(j)$ to be defined as zero when the drug level is below $v_{t h}$ and $\left(v_{d}(j)-v_{t h}\right)$ when $v(j) \geq v_{t h}$ using Big-M constraints as follows [21]:

$$
\begin{aligned}
v_{d}(j)-v_{t h} & \leq\left(v_{\max }-v_{t h}\right)(1-b(j)) \\
0(1-b(j)) & \leq \phi(j) \\
\left(v_{\max }-v_{t h}\right)(1-b(j)) & \geq \phi(j) \\
v_{t h}-v_{d}(j) & \leq v_{t h} b(j) \\
0 b(j) & \leq \phi(j)-\left(v_{d}(j)-v_{t h}\right) \\
v_{t h} b(j) & \geq \phi(j)-\left(v_{d}(j)-v_{t h}\right)
\end{aligned}
$$

Consider the situation when the drug plasma concentration is below the therapeutic value $\left(v_{d}(j) \leq v_{t h}\right)$. In this case, the left hand side of (14d) will be greater than zero which requires $b(j)$ to be one. This forces the left hand sides of both (14b) and (14c) to be zero, resulting in $\phi(j)$ being zero also. The left hand side of (14a) will be negative, so this constraint will be satisfied. The right hand side of constraints (14e) and (14f) will be positive on the range $\left[0, v_{t h}\right]$, so these constraints will also be satisfied.

Next consider the case where the drug is having an effect $\left(v_{d}(j) \geq v_{t h}\right)$. This forces $b(j)$ to be zero for the constraint (14a) to be satisfied. Thus, $\phi(j)$ will be forced to take on the value $v_{d}(j)-v_{t h}$ because the left hand side of constraints (14e) and (14f) will be zero. Furthermore $\phi(j)$ will exist on the range $\left[0,\left(v_{\max }-v_{t h}\right)\right]$ satisfying the constraints (14b) and (14c). Finally the the constraint (14d) will always be satisfied because the left hand side will have a maximum value of zero.

Using equation (13) and applying Euler's method, the discretized PD equation can be written as:

$$
P_{d}(j+1)=P_{d}(j)+h F\left(P_{d}(j), \phi(j)\right)
$$

3.2.4 Constraints: The toxicity constraints (5) can be transformed in a similar manner. The acute toxicity constraint can be replaced by a constraint on the maximum drug concentration at all points in the discretization as follows:

$$
v_{d}(j) \leq v_{\max } ; \quad \forall j \in[1, m]
$$

The integral in the exposure constraint (6) can be replaced with a summation representing integration using trapezoidal rule [20] in the following manner:

$$
\frac{h}{2} v_{d}(1)+h \sum_{j=2}^{m-1} v_{d}(j)+\frac{h}{2} v_{d}(m) \leq v_{\text {cum }}
$$

Furthermore the constraint on the maximum tumor size (8) can be written as:

$$
P_{d}(t) \leq \ln \left(N_{0}\right) ; \quad \forall j \in[3, m]
$$

One characteristic of discrete-time systems without direct feed through is that changes at a given step will not produce any output effect until the following timestep. Consequently, any effect afforded by drug administration will not be observed until two timesteps after administration. Because no drug administered at time zero can have an effect until the third timestep, and the cancer cells will continue to proliferate during these two timesteps, the constraint (8) was not enforced prior to the third timestep.

\section{Case Study}

\subsection{Parameters}

The parameters used in this study were taken from [1] and are given in Table 1. Table 1 contains three values

Table 1: Parameters used for the case study, with the exception of $h$, taken from Martin and Teo [1], where $[D]$ are the units of drug concentration.

\begin{tabular}{ccc}
\hline parameter & value & units \\
\hline \hline$\tau$ & 5 & months \\
$\theta$ & $10^{12}$ & cells \\
$N_{0}$ & $10^{10}$ & cells \\
$k_{d 1}$ & $2.7 \times 10^{-2}$ & days $^{-1}[D]^{-1}$ \\
$k_{d 2}$ & $8.4 \times 10^{-3}$ & days $^{-1}[D]^{-1}$ \\
$k_{d 3}$ & $1.5 \times 10^{-3}$ & days $^{-1}[D]^{-1}$ \\
$\gamma$ & .27 & days $^{-1}$ \\
$v_{t h}$ & 10 & {$[D]$} \\
$v_{\text {max }}$ & 50 & {$[D]$} \\
$v_{\text {cum }}$ & $4.1 \times 10^{3}$ & {$[D]$ days } \\
$t_{f}$ & 364 & days \\
$\Gamma$ & 0 & \\
$h$ & 0.5 & days \\
\hline
\end{tabular}

for $k_{d}$. The three values for $k_{d},\left(k_{d 1}, k_{d 2}\right.$, and $\left.k_{d 3}\right)$, represent the effects of three different drugs in order of decreasing efficacy, as considered in [1].

Because of the slow rate of elimination of the drug, $\gamma$, a significant amount of drug from a previous dose will still be present when the current dose is administered. This results in a time-varying lower bound on drug dose, with a minimum value $u_{\text {min }}=0$. By administering the drug directly into the plasma, the upper bound on the amount of drug that can be administered is limited by the acute toxicity constraint, (6) or (17). The maximum dose possible is also time-varying; therefore $u_{\max }=v_{\max }$ when no drug is present in the plasma or $u_{\max }$ is the amount of drug required to achieve a peak plasma concentration of $v_{\max }$. The timestep, $h$, selected was determined 
by the dynamics of the PK. The drug considered here has a very slow rate of clearance from the patient, so a timestep of 0.5 days can be used without sacrificing the accuracy of the drug PK.

\section{Results and Discussion}

The MILP was modeled in GAMS and solved using CPLEX on a dual Athlon MP 2200+ with 1GB of RAM. For the case of the most effective drug, $k_{d 1}$, the resulting problem had 8110 equations, 3693 continuous variables and 728 binary variables. The MILP was solved to optimality in 0.3 seconds with an objective function value of -30.4. This translates to a final cell population of less than one cell. Physiologically, this means all of the cancerous tissue would have been eliminated. This is the same objective function value $(J<1)$ reported by [1] for the $k_{d 1}$ case. The objective function values for the optimal control and MILP solution techniques are given in Table 2. The recommended drug administration profiles for the three different cases and the PD response for those profiles are shown in Figure 2.

Table 2: Objective function values for the MILP $\left(J_{M}\right)$ and optimal control $\left(J_{O}\right)$ solutions $[1]$.

\begin{tabular}{cccc}
\hline & $k_{d 1}$ & $k_{d 2}$ & $k_{d 3}$ \\
\hline \hline$J_{M}$ & $<1$ & $1.4 \times 10^{4}$ & $3.2 \times 10^{10}$ \\
$J_{O}$ & $<1$ & $1.2 \times 10^{3}$ & $1.8 \times 10^{9}$ \\
\hline
\end{tabular}

The solution presented here is qualitatively similar to the solution found using control parameterization techniques [1]. Because of the constraint on the maximum number of tumor cells, (8) and (18) for the continuous and discrete problems, respectively, there is a day-one dose administered in all cases. This brings the total number of cancer cells down rapidly. Subsequently, for the effective drug, $k_{d 1}$, no drug is administered until approximately the last half of the treatment cycle where the drug is administered to maintain circulating plasma concentrations near the maximum level, $v_{\max }$, while satisfying the cumulative toxicity constraints (17). For the less effective drugs, constants $k_{d 2}$ and $k_{d 3}$, the tumor population approaches the maximum requiring a larger number of drug doses earlier in the window. This is especially evident in the least effective case, $k_{d 3}$.

The MILP solutions are not only qualitatively similar to those found using optimal control. The differences found by the two techniques result from the discretization step size. By allowing the step size to approach zero, the optimal control solution is recovered. This was observed empirically by letting $h=.25, .1, .04$, which recovered solutions approaching those from [1] as $h$ decreased.

The MILP approach offers several advantages. The non-
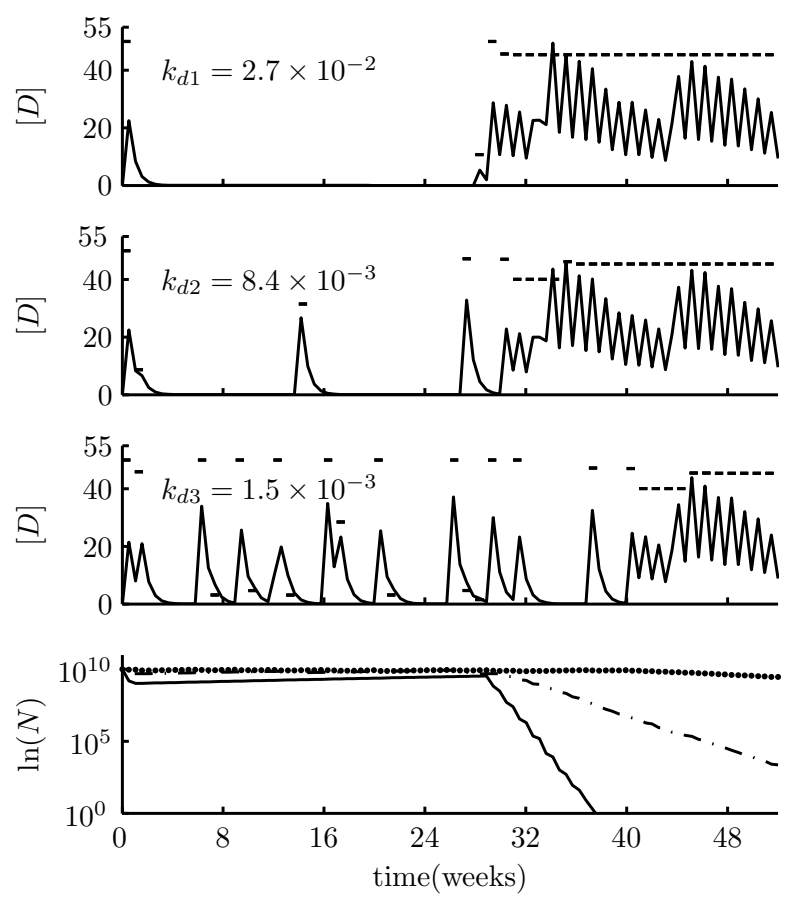

Figure 2: The top three panes represent the MILP dosing regimens predicted for the three different drug effects, $k$. The dosing levels are given by the solid horizontal bars and the drug concentration are given by the solid line. PD results for the three different values of $k$ are shown: $k_{d 1}=($ solid $), k_{d 2}=($ dash-dot $)$, and $k_{d 3}=($ dot $)$

linearities in this problem are easily easily handled by transforming the problem, thereby allowing the problem to be solved to optimality. This approach is also very flexible, allowing for clinically relevant constraints to easily be incorporated. Many clinical constraints can be more easily formulated in terms of integer and continuous variables. For example many drugs are administered orally in pill form. This results in a manipulated variable which takes on discrete values (e.g. no drug administered or a combination fixed amounts) [22]. Some drugs require a recovery period after several days of administration [23]. By adding a binary decision variable, which takes on the value of one when a drug is delivered and zero otherwise, this type of constraint formulates as a set of linear inequalities.

One concern with the formulation of the problem as addressed in [1], as well as this work, is the concept of the treatment cycle. Consider the current problem over the treatment window, where the solution is to deliver the drug as late as possible. While this might provide a mathematically optimal solution, the question of practicality must be addressed.

Clinicians consider problems in a moving horizon fashion with feedback provided at treatment cycles. The 
firs treatment cycle is used to establish the toxicity constraints for the patient. The second cycle determines if the treatment is efficacious. As a result, being able to formulate the problem in terms of a moving horizon optimization problem with feedback at the end of treatment cycles would be a more realistic approach.

\section{Summary}

A MILP approach has been proposed to address a class of chemotherapeutic dosing problems that have been considered in an optimal control perspective. The PD equations were logarithmically transformed, eliminating the nonlinearities associated with both tumor growth and drug effect. The work presented here is not only capable of reproducing the results obtained from optimal control but is readily adaptable to constraints commonly encountered by clinicians. Any algorithm to be implemented in a clinical setting would need to be adaptable in this manner.

\section{References}

[1] R. Martin and K. L. Teo. Optimal Control of Drug Administration in Cancer Chemotherapy. World Scientific, River Edge, NJ, 1994.

[2] K. Vermeulen, Z. N. Berneman, and D. R. Van Bockstaele. Cell cycle and apoptosis. Cell Prolif, 36:165$75,2003$.

[3] M. J. Boyer and I. F. Tannock. Cellular and Molecular Basis of Chemotherapy, chapter 15, pages 350-369. The Basic Science of Oncology. McGraw-Hill, New York, NY, 3rd edition, 1998.

[4] E. Reed. Cisplatin and Analogs, chapter 15, pages 466-481. Cancer Chemotherapy \& Biotherapy. Lippincott, Williams, \& Wilkins, Philadelphia, PA, 3rd edition, 2001.

[5] M. J. Egorin M.D. Personal communication. Feb 2003.

[6] R. Simon. Clinical Trials in Cancer, chapter 21, pages 521-538. Cancer: Principles \& Practice of Oncology. Lippincott, Williams, \& Wilkins, Philadelphia, PA, 6th edition, 2001.

[7] U. Ledzewicz and H. Schättler. Optimal bangbang controls for a two-compartment model in cancer chemotherapy. J. Optimization Theory Appl., 114(3):609-637, sept 2002.

[8] A. S. Matveev and A. V. Savkin. Application of optimal control theory to analysis of cancer chemotherapy regimens. Systems \& Control Letters, 46:311-321, 2002.

[9] R. B. Martin, M. E. Fisher, R. F. Minchin, and K. L. Teo. A mathematical model of cancer chemotherapy with an optimal selection of parameters. Math. Biosci., 99:205-230, 1990.
[10] J. Smieja and A. Swierniak. Optimal control of certain infine dimensional systems with application to chemotherapy modelling. In Proc. American Control Conf., pages 3466-3471, Anchorage, AK, May 2002. ACC.

[11] F. L. Pereira, C. E. Pedreira, M. R. Pinho, M. H. Fernandes, and J. B. Sousa. An optimal control algorithm for multidrug cancer chemotherapy design. In Proc. IEEE EMBS Ann. Conf., volume 12, pages 10211022, 1990.

[12] J. H. Lee. Modeling and identification for nonlinear predictive control: Requirements, current status and future research needs. In International Symposium on Nonlinear Model Predictive Control: Assessment and Future Directions, pages 91-107, Ascona, Switzerland, 1998.

[13] M. Morari and E. Zafiriou. Robust Process Control. Prentice-Hall, Englewood Cliffs, NJ, 1989.

[14] Ewart Carson and Claudio Cobelli, editors. Modelling Meghodology for Physiology and Medicine. Academic Press, Harcourt Place, 32 Jamestown Road, 2001.

[15] A. Asachenkov, G. Marchuk, R. Mohler, and S. Zuev. Disease Dynamics. Birkhäuser, Boston, 1994.

[16] J. M. Slingerland and I. F. Tannock. Cell Proliferation and Cell Death, chapter 7, pages 134-165. The Basic Science of Oncology. McGraw-Hill, third edition, 1998.

[17] L. Norton. A Gompertzian model of human breast cancer growth. Cancer Res., 48:7067-7071, 1988.

[18] J. C. Panetta and J. Adam. A mathematical model of cycle-specific chemotherapy. Math. Comput. Model., $22(2): 67-82,1995$.

[19] C. T. Chen. Linear System Theory and Design. Holt, Rinehart, and Winston, Austin, TX, 1984.

[20] E. Kreyszig. Advanced Engineering Mathematics. John Wiley \& Sons, New York, NY, 8th edition, 1999.

[21] M. L. Tyler and M. Morari. Propositional logic in control and monitoring problems. Automatica, 35:565$582,1999$.

[22] Merrill J. Egorin. Overview of recent topics in clinical pharmacology of anticancer agents. Cancer Chemother. Pharmacol. Suppl., 42:S22-S30, 1998.

[23] K. D. Tew, G. M. Colvin, and B. A. Chabner. Alkylating Agents, chapter 13, pages 373-414. Cancer Chemotherapy \& Biology. Lippincott Williams \& Wilkins, 0781722691, 3rd edition, 2001. 